\title{
The Impact of Accidental Hypothermia on Mortality in Trauma Patients Overall and Patients with Traumatic Brain Injury Specifically: A Systematic Review and Meta-Analysis
}

\author{
David Rösli $^{1}$ Beat Schnüriger ${ }^{1}$ Daniel Candinas ${ }^{1} \cdot$ Tobias Haltmeier $^{1}$
}

Accepted: 1 August 2020/Published online: 28 August 2020

(C) The Author(s) 2020

\begin{abstract}
Background Accidental hypothermia is a known predictor for worse outcomes in trauma patients, but has not been comprehensively assessed in a meta-analysis so far. The aim of this systematic review and meta-analysis was to investigate the impact of accidental hypothermia on mortality in trauma patients overall and patients with traumatic brain injury (TBI) specifically.

Methods This is a systematic review and meta-analysis using the Ovid Medline/PubMed database. Scientific articles reporting accidental hypothermia and its impact on outcomes in trauma patients were included in qualitative synthesis. Studies that compared the effect of hypothermia vs. normothermia at hospital admission on in-hospital mortality were included in two meta-analyses on (1) trauma patients overall and (2) patients with TBI specifically. Meta-analysis was performed using a Mantel-Haenszel random-effects model.

Results Literature search revealed 264 articles. Of these, 14 studies published 1987-2018 were included in the qualitative synthesis. Seven studies qualified for meta-analysis on trauma patients overall and three studies for metaanalysis on patients with TBI specifically. Accidental hypothermia at admission was associated with significantly higher mortality both in trauma patients overall (OR 5.18 [95\% CI 2.61-10.28]) and patients with TBI specifically (OR 2.38 [95\% CI 1.53-3.69]).

Conclusions In the current meta-analysis, accidental hypothermia was strongly associated with higher in-hospital mortality both in trauma patients overall and patients with TBI specifically. These findings underscore the importance of measures to avoid accidental hypothermia in the prehospital care of trauma patients.
\end{abstract}

Electronic supplementary material The online version of this article (https://doi.org/10.1007/s00268-020-05750-5) contains supplementary material, which is available to authorized users.

Congress presentation: Preliminary data have been presented at the 20th European Congress of Trauma and Emergency Surgery (ECTES), May 5, 2019, Prague, Czech Republic.

Tobias Haltmeier

tobias.haltmeier@icloud.com

David Rösli

david.roesli@outlook.de

Beat Schnüriger

beat.schnueriger@insel.ch

\section{Background}

Accidental hypothermia occurs frequently in trauma patients. Up to $66 \%$ of patients being admitted to the emergency department (ED) for major trauma have been

\section{Daniel Candinas}

daniel.candinas@insel.ch

1 Department of Visceral Surgery and Medicine, Inselspital, Bern University Hospital, University of Bern, Bern, Switzerland 
reported to suffer from accidental hypothermia [1]. Hypothermia is commonly defined as a core body temperature $<35{ }^{\circ} \mathrm{C}$ and further classified into mild $\left(35^{\circ} \mathrm{C}-\right.$ $\left.32{ }^{\circ} \mathrm{C}\right)$, moderate $\left(32{ }^{\circ} \mathrm{C}-28{ }^{\circ} \mathrm{C}\right)$, and severe $\left(<28^{\circ} \mathrm{C}\right)[2]$. The etiology is multifactorial, comprising environmental exposure, hemorrhagic shock, not sufficiently prewarmed solutions for volume resuscitation and drugs causing reduced vasoconstriction and a higher shivering threshold, such as anesthetics and muscle relaxants [3-5].

Accidental hypothermia has been described as a predictor for worse outcomes, including higher mortality, higher blood product transfusion requirements, and longer intensive care unit (ICU) and hospital length of stay (LOS), both in trauma patients overall and patients with traumatic brain injury (TBI) specifically [6-10]. In severely injured trauma patients, mortality increases with the degree of hypothermia [9].

On the other hand, potential beneficial effects of hypothermia have been discussed in trauma patients, too [11-13]. In analogy to the targeted temperature management in patients with cardiac arrest, the rationale for induced hypothermia in trauma patients with TBI is to decrease the deleterious effects of secondary brain injury. Hypothermia is thought to reduce the effects of secondary brain injury through multiple mechanisms, including reduced excitotoxicity, oxidative stress, apoptosis, autophagy, and inflammation [14, 15]. Experimental studies suggest that mild hypothermia may alter the inflammatory response after TBI [16, 17]. Furthermore, animal models revealed reduced mortality, an improved behavioral outcome, and a diminished disruption of the blood-brain barrier in animals subjected to mild therapeutic hypothermia after having suffered from TBI $[18,19]$.

Although accidental hypothermia is important not only in the clinical management of trauma patients, but also in traumatic brain injury research, it has not yet been comprehensively addressed in a systematic literature review and meta-analysis. The aim of this literature review and meta-analysis was to assess the effect of accidental hypothermia on outcomes in trauma patients overall and patients with TBI specifically. We hypothesized that accidental hypothermia has a strong adverse impact on outcomes both in trauma patients overall and patients with TBI specifically.

\section{Methods}

This is a systematic literature review and meta-analysis exploring the impact of accidental hypothermia on outcomes in trauma patients overall and patients with TBI specifically.
PRISMA 2009 Guidelines [20, 21] were followed throughout the systematic literature review, reporting of the data, and discussion (Supplemental Table 1).

Articles were assessed, and data were extracted by two reviewers (DR, TH). Differences were resolved by consensus.

This study has been registered at ClinicalTrials.gov (ID NCT04332237).

\section{Literature search}

A systematic literature search was performed using Ovid Medline [22] and PubMed [23] (Medline database, US National Library of Medicine). The search strategy was based on the PICOS strategy [24, 25]. Medical Subject Headings $(\mathrm{MeSH})$ were used as search terms whenever feasible [26]. No additional filters were utilized. Literature search was conducted in August 2019.

Literature search was performed including the following search terms:

(multiple trauma OR polytrauma OR major trauma OR ISS OR APACHE) AND (hypothermia OR accidental hypothermia) AND (mortality OR intensive care unit OR survival rate OR length of stay OR outcome OR bleeding OR ventilator).

All abstracts were screened for eligibility. Articles were included in the qualitative synthesis if they met the following criteria:

1. Study in trauma patients.

2. Reported body temperature and time of measurement.

3. Reported impact of accidental hypothermia on outcomes.

References relevant to the topic listed in the bibliography of articles found through the search strategy mentioned above were also included. Only original research articles in English language were considered for inclusion. Articles with no relevance to the topic, case reports, systematic reviews, and articles covering therapeutic hypothermia were excluded. Full text articles of abstracts that met the inclusion criteria were subsequently assessed.

If two or more studies acquired data from the same database during an overlapping time period and included the same patient population, the study covering the longest time period or study that qualified for meta-analysis was selected for inclusion. The other studies were excluded from the systematic review and meta-analysis.

\section{Endpoint}

The endpoint investigated in the current systematic review and meta-analysis was in-hospital mortality. In studies that reported overall mortality or mortality not further specified, 
the reported mortality rate was assumed to correspond to in-hospital mortality.

\section{Meta-analysis}

Two meta-analyses were preformed including (1) trauma patients overall and (2) patients with TBI specifically. Studies that reported in-hospital mortality of hypothermic and normothermic trauma patients overall or patients with TBI specifically, based on the temperature measured at ED admission, were included in meta-analyses. The number of survivors and non-survivors in the hypothermic and normothermic group was extracted from these studies. For studies that reported mortality of hypothermic and normothermic patients as percentages only, the actual number of patients was calculated from the percentages.

Meta-analysis was conducted using a Mantel-Haenszel random-effects model. The estimated effect size for mortality was reported as odds ratio (OR) and $95 \%$ confidence interval (CI) for each study as well as for the overall cohort. Statistical heterogeneity of the studies included was measured using Cochrane Q statistics and I-square [27, 28] and interpreted based on the current consensus-based recommendations by Gagnier et al. [29].

Sensitivity analysis was performed by repeating the Mantel-Haenszel random-effects model in the subgroups of studies using the same definition of hypothermia, studies with similar inclusion criteria regarding the ISS, studies published during the last 10 years, and studies including more than 10,000 patients.

Statistical analysis was performed using Review Manager (RevMan) Version 5.3. (Copenhagen: The Nordic Cochrane Collaboration, 2014).

\section{Quality assessment}

The quality of the studies incorporated in the systematic literature review and meta-analysis was assessed using the Newcastle-Ottawa Scale (NOS) for cohort studies [30] (Supplemental Table 2).

\section{Results}

\section{Study selection}

A total of 251 articles were obtained from Medline. Similar search results were obtained using PubMed. Fourteen additional publications cited in these 251 articles were found to be relevant to the topic, too. After removal of duplicates, abstracts of 264 articles were screened for eligibility. Abstracts of twenty-four studies met the inclusion criteria. Of these, the full text articles were assessed (Fig. 1).

Ten studies reported data from the same database or registry during an overlapping time period [6-8, 10, 31-36]. Of these, three studies were excluded from the review and meta-analysis [10, 33, 34]. Three other studies included patients suffering from TBI specifically. These three studies were excluded from meta-analysis for trauma patients overall, but included in meta-analysis for patients with TBI specifically $[6,7,36]$.

Altogether, after the assessment of the full text articles, a total of ten studies were excluded and 14 studies were included in qualitative synthesis.

\section{Study characteristics}

Study characteristics are outlined in Table 1. Publication years ranged from 1987 to 2018 . Eleven studies were retrospective analyses [6-8, 31, 32, 35-40], and three studies were designed prospectively [1, 41, 42].

\section{Traumatic brain injury}

Four studies included patients with severe traumatic brain injury and accidental hypothermia specifically. The study by Bukur et al. included patients with isolated TBI [36], while the other three studies by Rubiano et al., Tohme et al., and Winkelmann et al. were comprised of patients suffering from concomitant TBI, i.e., patients with extracranial injuries were not excluded [6, 7, 42].

\section{Definition of hypothermia and temperature measurement}

Definitions of hypothermia used in the studies included are outlined in Table 1. Temperature measurement methods were specified in six studies and comprised nasopharyngeal [39], esophageal [1, 7, 31, 32, 41], bladder [7, 31, 32], rectal [32, 39], tympanic [32, 41], axillary [32], and oral [32] temperature probes. Eight studies did not describe the exact method of temperature measurement $[6,8,35-38,40,42]$.

\section{Patient characteristics}

Patient characteristics are summarized in Table 2. Included patients were predominantly male (range 71-79\%) [6, 31, 35, 36, 39, 41, 42]. Eight studies compared the age of the normothermic and hypothermic patient groups [7, 8, 31, 35-37, 39, 40]. In the studies by Bukur et al. and Martin et al., hypothermic patients were significantly older than normothermic patients $[8,35]$. In the other six studies, 
Fig. 1 PRISMA 2009 flow diagram
Records identified through database
searching
$(\mathrm{n}=251)$
Additional records identified

through found articles $(n=14)$

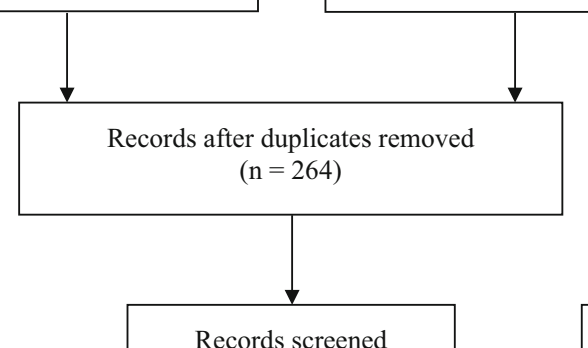

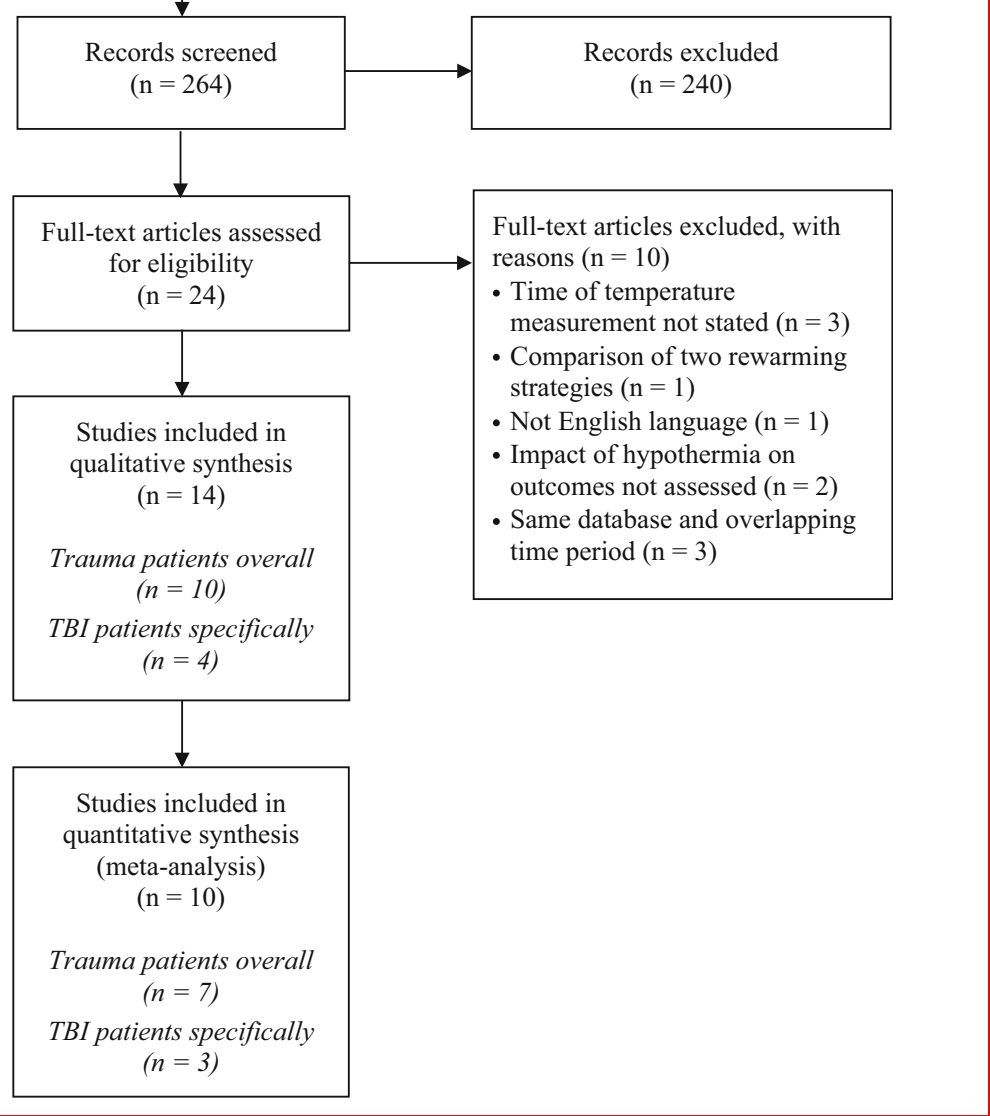

no significant association of age and hypothermia was found [7, 31, 36, 37, 39, 40].

Eight studies assessed the injury severity in normothermic and hypothermic patients separately. In seven studies, a significantly higher ISS was found in hypothermic compared to normothermic patients $[7,8,31,35,36,39,40]$. In the study by Wang et al., the proportion of patients with higher New Injury Severity Scores (NISS) was higher in the hypothermic compared to the normothermic group [32].

In the twelve studies that reported temperature at ED admission, the proportion of hypothermic patients ranged from 1.6 to $44.6 \%$ [6-8, 31, 32, 35-38, 40-42].

\section{Mortality}

Four studies specifically reported in-hospital mortality [32, 35, 37, 40]. Balvers et al. reported both the 24-h and 28-day mortality rate [39]. Tohme et al. described 14-day mortality [42]. Eight studies reported overall mortality or mortality without further specification [1, 6-8, 31, 36, 38, 41].

In seven studies, death rates were significantly higher in hypothermic trauma patients compared to normothermic patients [8, 31, 35, 37, 39-41]. Bukur et al., Rubiano et al., Tohme et al., and Winkelmann et al., in their cohorts consisting of patients with TBI specifically, observed a significantly higher mortality rate when accidental 


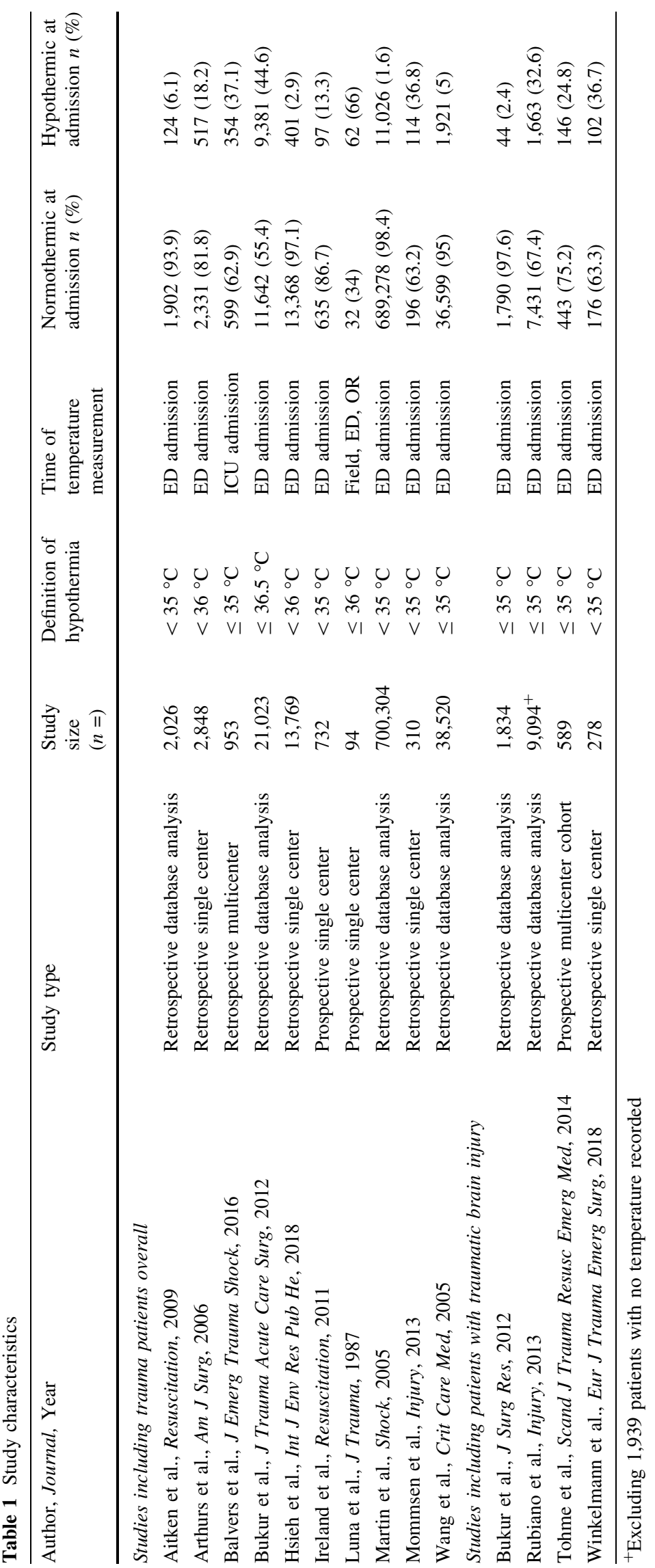




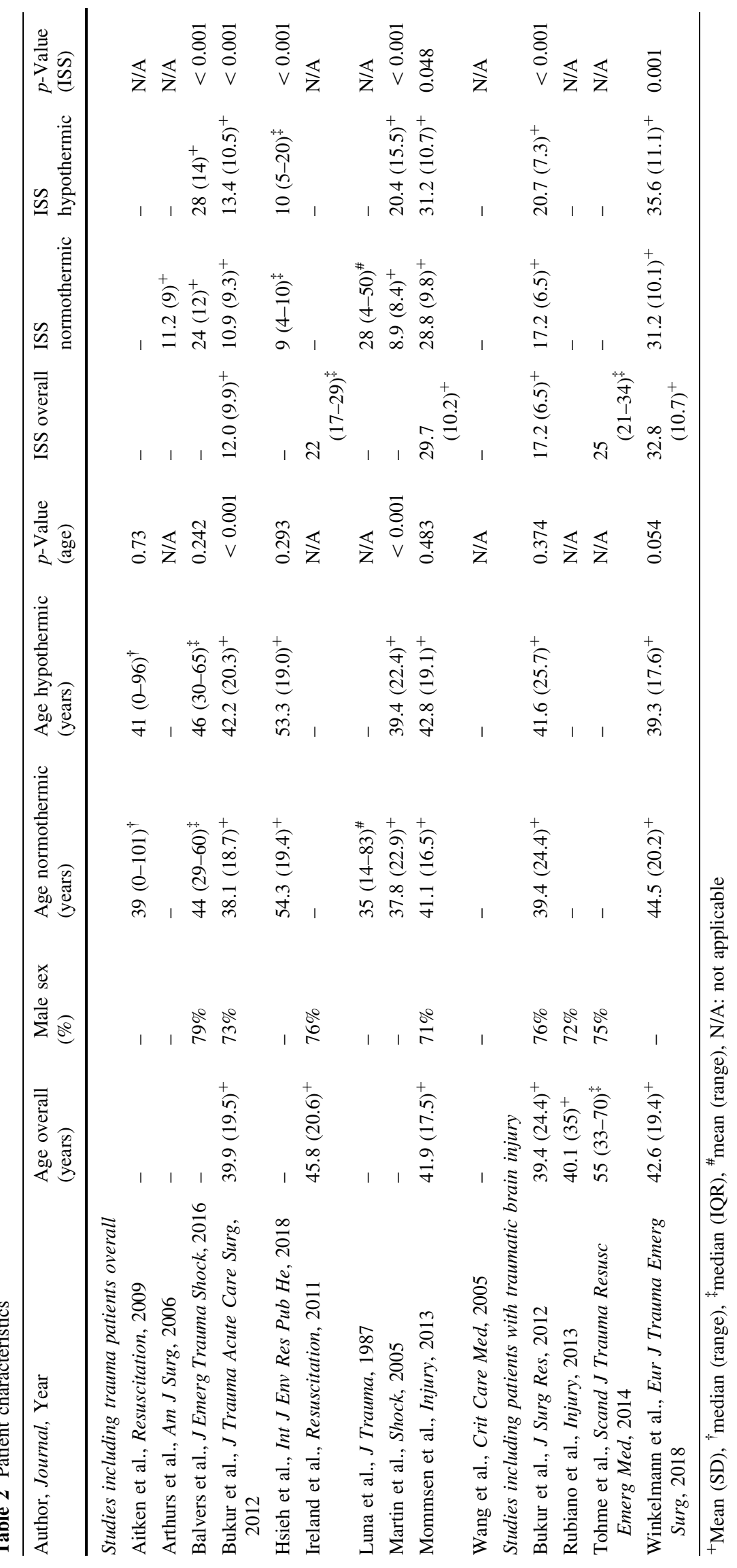


hypothermia was present at hospital admission $[6,7,36,42]$.

In thirteen studies, the effect of hypothermia on mortality was adjusted for various covariates, including the injury severity. Eleven studies adjusted for the ISS $[7,8,31,35-42]$ and two studies for the NISS [6, 32] in multivariable analysis for mortality. In eleven studies, hypothermia was reported as an independent predictor for mortality, even after adjustment for the injury severity and other covariates [6, 8, 32, 35-42]. In two studies, the effect of hypothermia on mortality did not remain significant after adjustment in multivariable analysis [7, 31] (Table 3).

\section{Meta-analysis for mortality}

Seven studies comparing in-hospital mortality of normothermic and hypothermic trauma patients at ED admission were included in meta-analysis for trauma patients overall [8, 31, 32, 35, 37, 40, 41]. Meta-analysis for trauma patients overall revealed a significantly higher mortality in patients with hypothermia on admission (OR 5.18 [95\% CI 2.61-10.28]) (Fig. 2).

Three studies were included in meta-analysis for patients with TBI specifically [6, 7, 36]. Meta-analysis for patients with TBI specifically revealed significantly higher mortality in hypothermic compared to normothermic patients (OR 2.38 [95\% CI 1.53-3.69]) (Fig. 2).

In sensitivity analysis including studies with the same definition of hypothermia (temperature $<35^{\circ} \mathrm{C}$, four studies, 703,372 patients [8, 31, 37, 41]), studies with similar patient inclusion criteria regarding the ISS (ISS $\geq 16$, three studies, 3,068 patients [31, 37, 41]), studies published during the last 10 years (four studies, 35,834 patients $[31,35,40,41])$, and studies with more than 10,000 patients (four studies, 773,616 patients $[8,32,35,40])$, hypothermia on admission was associated with significantly higher mortality, too (OR 6.15 [95\%CI 3.43-11.05], $I^{2}=90 \%$; OR 5.02 [95\%CI 3.35-7.52], $I^{2}=39 \%$; OR 3.76 [95\%CI 1.48-9.52], $I^{2}=96 \%$; and OR 5.55 [95\%CI 2.23-3.84], $I^{2}=100 \%$ ).

\section{Blood product transfusion and coagulation disorder}

Transfused blood products in normothermic and hypothermic trauma victims were explored by four studies [7, 31, 38, 41]. In two studies, packed red blood cells (PRBCs) transfusion rates were significantly higher in hypothermic patients [7, 31]. In the other two studies, no significant difference in PRBC transfusions between hypothermic and normothermic patients was found [38, 41].

Mommsen et al. and Winkelmann et al. reported significantly higher fresh frozen plasma (FFP) and platelet
(PLT) transfusion rates in hypothermic trauma patients [7, 31].

INR values were provided by Balvers et al. and Ireland et al. and were significantly higher in hypothermic individuals [39, 41] (Table 3 ).

\section{Quality assessment}

Overall, the quality of the studies included was satisfactory. Twelve studies received nine out of nine stars, one study received eight stars, and one study seven stars (Supplemental Table 2).

\section{Discussion}

The aim of this literature review and meta-analysis was to systematically assess the impact of accidental hypothermia on outcomes in trauma patients overall and in patients with TBI specifically. Meta-analysis revealed accidental hypothermia as a significant predictor for higher in-hospital mortality both in trauma patients overall and patients with TBI specifically. With an overall OR of 5.18 for trauma patients and 2.38 for patients with TBI specifically, hypothermia had a strong impact on mortality.

Taking into account the strong impact of accidental hypothermia on mortality, efforts should be made to prevent hypothermia in trauma patients whenever possible. Persistent hypothermia in severely injured trauma patients decreases the likelihood of successful resuscitation [43]. Consequently, it must be prevented in the prehospital setting, but also during early hospital care. Measures to avoid and treat hypothermia include the removal of wet clothing, a warm environment, warming blankets, warm parenteral fluids including blood products, avoidance of unnecessary anesthesia, avoidance of prolonged surgery with an open abdomen, warm thoracic or abdominal lavage, and, in cases of severe hypothermia with cardiac instability or arrest, rewarming with extracorporeal membrane oxygenation or cardiopulmonary bypass [2, 44-46]. These techniques to avoid hypothermia in trauma patients and combat casualties are part of the current Advanced Trauma Life Support [47] and Tactical Combat Casualty Care [48] guidelines.

Trauma-induced coagulopathy is frequent in severely injured patients and has been shown to be associated with higher mortality [49, 50]. Hypothermia, acidosis, and hemodilution due to volume resuscitation share complex interactions and influence each other disadvantageously, leading to an aggravation of coagulopathy in trauma patients [51, 52].

In the current review, two studies reported significantly higher transfusion requirements [7, 31] and two other 


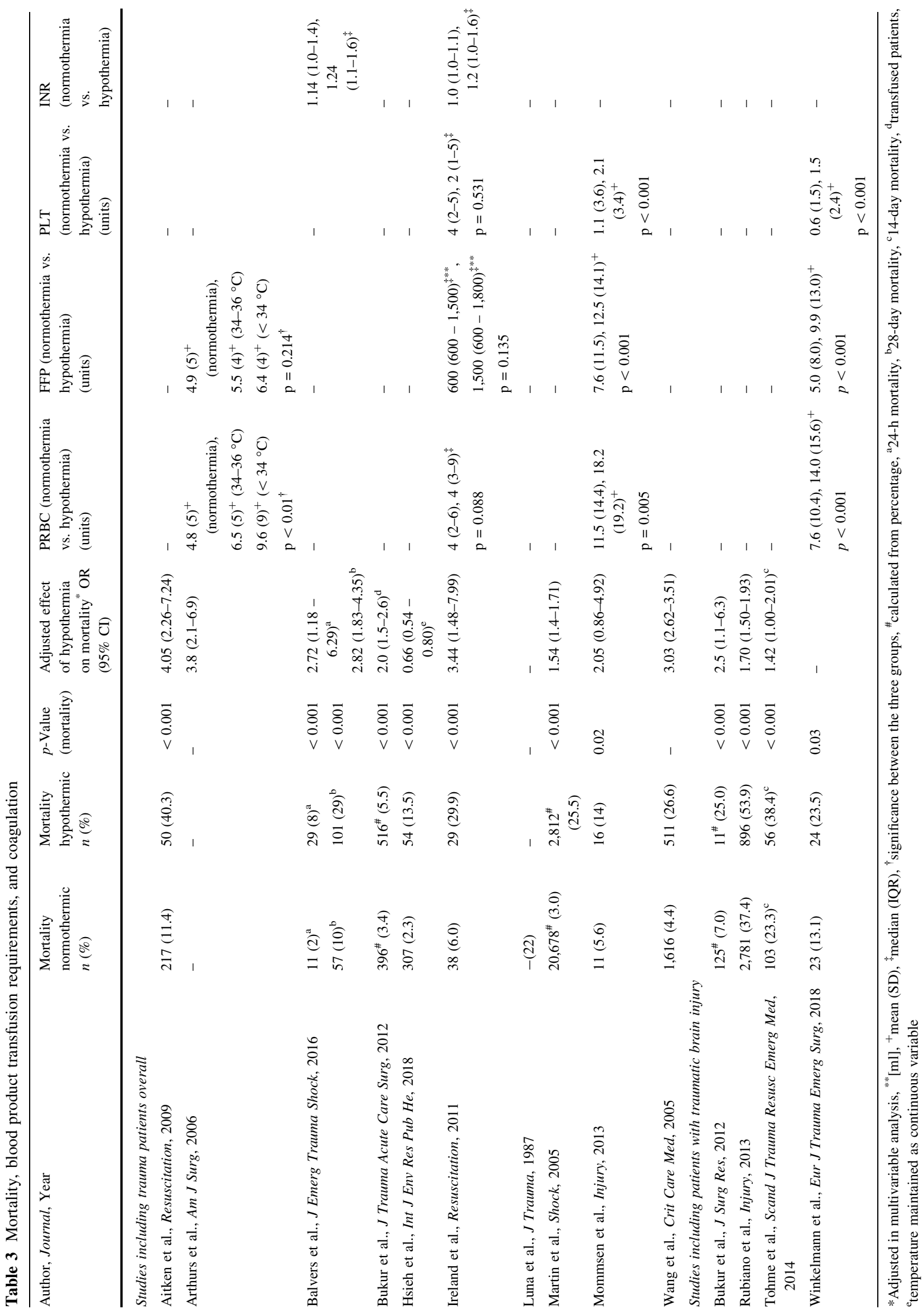




\section{Effect of hypothermia on mortality in trauma patients}

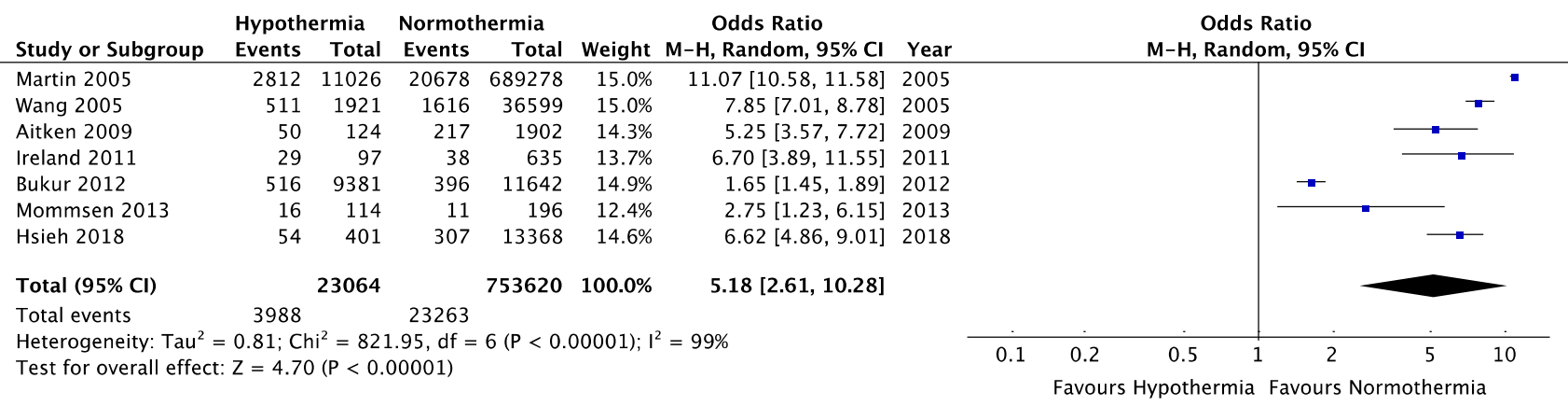

\section{Effect of hypothermia on mortality in trauma patients with TBI}

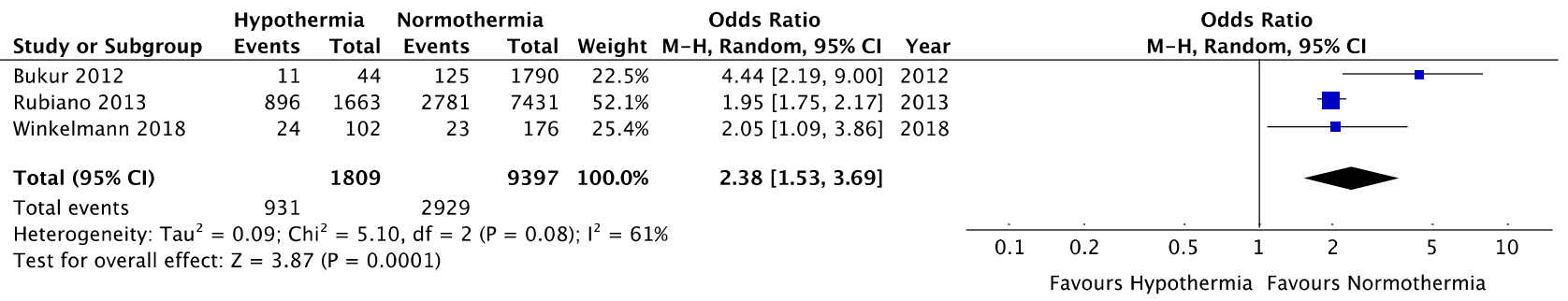

Fig. 2 Meta-analysis

studies significantly higher INR values [39, 41] in hypothermic patients compared to normothermic patients. In the setting of severely injured trauma patients, higher transfusion requirements and INR values may serve as surrogate markers for coagulopathy. Thus, the higher mortality rate in patients suffering from accidental hypothermia in the studies included in this review may be related to hypothermia-induced coagulopathy.

However, as in the studies included the effect of hypothermia on transfusion requirements and the INR was not adjusted for other clinically important factors, such as the injury severity and transport time, these results may be confounded.

In this systematic review, three studies that included patients with TBI specifically reported a higher mortality rate in hypothermic compared to normothermic patients $[6,36,42]$. Of note, all three studies investigated patients with severe TBI, but only one included patients with isolated severe TBI [36]. Any conclusion concerning the effect of hypothermia on mortality in patients suffering from isolated TBI and mild or moderate TBI is therefore limited.

Beneficial effects of hypothermia on secondary brain injury have previously been discussed in the literature [11-13]. Hypothermia leads to the suppression of multiple damaging mechanisms in the injured brain, including excitotoxicity, oxidative stress, apoptosis, autophagy, and inflammation [14, 15]. Furthermore, experimental studies suggest that hypothermia is associated with a decreased blood-brain barrier permeability and inhibition of diffuse axonal injury [18, 53].

However, these potential neuroprotective effects of hypothermia in traumatic brain injury are most likely outweighed by the deleterious effect of the trauma-induced coagulopathy and consecutive aggravated intracranial bleeding [11, 54].

Hypothermia due to hypothalamic dysfunction has been described in the literature [55] and may have potentially contributed to the higher mortality in hypothermic patients with severe TBI. However, hypothermia in patients with hypothalamic dysfunction would not be expected at ED admission, but rather later in the hospital course.

Further research on the effect of hypothermia in patients suffering from TBI, especially those with isolated brain injury, is warranted.

In the existing literature, two other meta-analyses by Harris et al. and McHugh et al. reported hypothermia in patients with traumatic brain injury [56, 57]. However, the effect of accidental hypothermia at ED admission was not addressed in these studies, and none of the studies included in these meta-analyses met the inclusion criteria for the current analysis. Harris et al. included seven randomized controlled trials comparing the effect of induced hypothermia versus normothermia in patients with 
posttraumatic head injury on several endpoints, including the Glasgow Outcome Scale Score, intracranial pressure, pneumonia, cardiac arrhythmia, prothrombin time, and partial thromboplastin time. No benefit of therapeutic hypothermia on these endpoints was shown [57]. The metaanalysis by McHugh et al. [56] incorporated ten studies from the International Mission for Prognosis And Clinical Trial (IMPACT) database [58]. The main outcome was the Glasgow Outcome Scale Score at 6 months in patients having suffered from moderate or severe TBI. In this metaanalysis, hypothermia was associated with adverse outcomes, too [56].

This systematic review and meta-analysis has several limitations.

First, eleven studies were designed retrospectively [6-8, 31, 32, 35-40], and only three studies prospectively [1, 41, 42]. Thus, most studies included had the known limitations of retrospective analyses.

Second, substantial statistical heterogeneity was found both in meta-analysis on trauma patients overall and patients with TBI specifically. Statistical heterogeneity of the current meta-analysis may be explained by methodological and clinical heterogeneity of the studies included, i.e., different definitions of hypothermia and different temperature measurement methods, as well as differences in patient characteristics, including sex, age, and injury severity. Accordingly, sensitivity analysis of the subgroup of studies with similar inclusion criteria regarding the ISS revealed lower heterogeneity. However, as hypothermia had a strong impact on mortality in all studies included and the subgroups assessed in sensitivity analysis, heterogeneity did not relevantly affect the finding of this metaanalysis.

Third, the effect of hypothermia on mortality may have been confounded by the higher injury severity, deranged physiology, and higher transfusion requirements in hypothermic patients. However, eleven of the studies included revealed hypothermia as an independent predictor for higher mortality, even after adjustment for the injury severity and various covariates in multivariable analysis $[6,8,32,35-42]$, including two studies that adjusted for blood product transfusions [38, 39]. Furthermore, as prehospital blood product transfusion is rare, a confounding of the effect of hypothermia on mortality by transfusion is unlikely in the current meta-analysis that included patients with temperature measurement at ED admission only.

Fourth, an estimation of the degree of hypothermia of the included patient cohorts is difficult, as only two studies reported the mean or median temperature $[1,36]$.

In conclusion, in the current systematic review and meta-analysis, accidental hypothermia was strongly associated with significantly higher in-hospital mortality both in trauma patients overall and patients with TBI specifically. These findings underscore the importance of measures to avoid accidental hypothermia in the prehospital care of trauma patients.

Funding Open access funding provided by University of Bern.

\section{Compliance with ethical standards}

Disclosure David Rösli, Beat Schnüriger, Daniel Candinas, and Tobias Haltmeier have no conflicts of interest or financial ties to disclose.

Open Access This article is licensed under a Creative Commons Attribution 4.0 International License, which permits use, sharing, adaptation, distribution and reproduction in any medium or format, as long as you give appropriate credit to the original author(s) and the source, provide a link to the Creative Commons licence, and indicate if changes were made. The images or other third party material in this article are included in the article's Creative Commons licence, unless indicated otherwise in a credit line to the material. If material is not included in the article's Creative Commons licence and your intended use is not permitted by statutory regulation or exceeds the permitted use, you will need to obtain permission directly from the copyright holder. To view a copy of this licence, visit http://creativecommons. org/licenses/by/4.0/.

\section{References}

1. Luna GK, Maier RV, Pavlin EG et al (1987) Incidence and effect of hypothermia in seriously injured patients. J Trauma 27:1014-1018

2. Accidental hypothermia in adults - UpToDate, 2019. Available from: https://www.uptodate.com/contents/accidental-hypother mia-in-adults?topicRef $=13854 \&$ source $=$ related_link

3. Hohlrieder M, Kaufmann M, Moritz M et al (2007) Management of accidental hypothermia. Anaesthesist 56:805-811

4. Seekamp A, van Griensven M, Hildebrandt F et al (1999) Adenosine-triphosphate in trauma-related and elective hypothermia. J Trauma 47:673-683

5. Gentilello LM (1995) Advances in the management of hypothermia. Surg Clin North Am 75:243-256

6. Rubiano AM, Sanchez AI, Estebanez G et al (2013) The effect of admission spontaneous hypothermia on patients with severe traumatic brain injury. Injury 44:1219-1225

7. Winkelmann M, Soechtig W, Macke C et al (2018) Accidental hypothermia as an independent risk factor of poor neurological outcome in older multiply injured patients with severe traumatic brain injury: a matched pair analysis. Eur J Trauma Emerg Surg 09:09

8. Martin RS, Kilgo PD, Miller PR et al (2005) Injury-associated hypothermia: an analysis of the 2004 National Trauma Data Bank. Shock 24:114-118

9. Jurkovich GJ, Greiser WB, Luterman A et al (1987) Hypothermia in trauma victims: an ominous predictor of survival. J Trauma 27:1019-1024

10. Shafi S, Elliott AC, Gentilello L (2005) Is hypothermia simply a marker of shock and injury severity or an independent risk factor for mortality in trauma patients? Analysis of a large national trauma registry. J Trauma 59:1081-1085 
11. Marion DW, Penrod LE, Kelsey SF et al (1997) Treatment of traumatic brain injury with moderate hypothermia. N Engl J Med 336:540-546

12. Holzer M (2002) Mild therapeutic hypothermia to improve the neurologic outcome after cardiac arrest. $\mathrm{N}$ Engl $\mathrm{J}$ Med 346:549-556

13. Saad H, Aladawy M (2013) Temperature management in cardiac surgery. Glob Cardiol Sci Pract 2013:44-62

14. Lee JH, Zhang J, Yu SP (2017) Neuroprotective mechanisms and translational potential of therapeutic hypothermia in the treatment of ischemic stroke. Neural Regen Res 12:341-350

15. Fritz HG, Bauer R (2004) Secondary injuries in brain trauma effects of hypothermia. J Neurosurg Anesthesiol 16(1):43-52

16. Truettner JS, Suzuki T, Dietrich WD (2005) The effect of therapeutic hypothermia on the expression of inflammatory response genes following moderate traumatic brain injury in the rat. Brain Res Mol Brain Res 138:124-134

17. Zhang F, Dong H, Lv T et al (2018) Moderate hypothermia inhibits microglial activation after traumatic brain injury by modulating autophagy/apoptosis and the MyD88-dependent TLR4 signaling pathway. J Neuroinflammation 15:273

18. Jiang JY, Lyeth BG, Kapasi MZ et al (1992) Moderate hypothermia reduces blood-brain barrier disruption following traumatic brain injury in the rat. Acta Neuropathol 84:495-500

19. Clifton GL, Jiang JY, Lyeth BG et al (1991) Marked protection by moderate hypothermia after experimental traumatic brain injury. J Cereb Blood Flow Metab 11:114-121

20. Moher D, Liberati A, Tetzlaff J et al (2009) Preferred reporting items for systematic reviews and meta-analyses: the PRISMA statement. PLoS Med 6:e1000097

21. PRISMA, 2015, Available from: https://prisma-statement.org/ Default.aspx

22. Ovid Medline, 2018, Available from: https://www.ovid.com/site/ catalog/databases/901.jsp

23. Medicine USNLo. PubMed., 2018, Available from: https://www. ncbi.nlm.nih.gov/pubmed/

24. Cochrane Handbook for Systematic Reviews of Interventions, 2011, Available from: https://training.cochrane.org/handbook

25. Methley AM, Campbell S, Chew-Graham C et al (2014) PICO, PICOS and SPIDER: a comparison study of specificity and sensitivity in three search tools for qualitative systematic reviews. BMC Health Serv Res 14:579

26. MeSH, 2018, Available from: https://www.ncbi.nlm.nih.gov/ mesh

27. Sedgwick P (2015) Meta-analyses: what is heterogeneity? BMJ 350:h1435

28. How to interpret results of meta-analysis (Version 1.3), 2018, Available from: https://repub.eur.nl/pub/80102

29. Gagnier JJ, Morgenstern H, Altman DG et al (2013) Consensusbased recommendations for investigating clinical heterogeneity in systematic reviews. BMC Med Res Methodol 13:106

30. Newcastle-Ottawa Scale (NOS), 2018, Available from: https:// www.ohri.ca/Programs/clinical_epidemiology/oxford.asp

31. Mommsen P, Andruszkow H, Fromke C et al (2013) Effects of accidental hypothermia on posttraumatic complications and outcome in multiple trauma patients. Injury 44:86-90

32. Wang HE, Callaway CW, Peitzman AB et al (2005) Admission hypothermia and outcome after major trauma. Crit Care Med 33:1296-1301

33. Konstantinidis A, Inaba K, Dubose J et al (2011) The impact of nontherapeutic hypothermia on outcomes after severe traumatic brain injury. J Trauma 71:1627-1631

34. Inaba K, Teixeira PG, Rhee P et al (2009) Mortality impact of hypothermia after cavitary explorations in trauma. World J Surg 33:864-869
35. Bukur M, Hadjibashi AA, Ley EJ et al (2012) Impact of prehospital hypothermia on transfusion requirements and outcomes. J Trauma Acute Care Surg 73:1195-1201

36. Bukur M, Kurtovic S, Berry C et al (2012) Pre-hospital hypothermia is not associated with increased survival after traumatic brain injury. J Surg Res 175:24-29

37. Aitken LM, Hendrikz JK, Dulhunty JM et al (2009) Hypothermia and associated outcomes in seriously injured trauma patients in a predominantly sub-tropical climate. Resuscitation 80:217-223

38. Arthurs Z, Cuadrado D, Beekley A et al (2006) The impact of hypothermia on trauma care at the 31 st combat support hospital. Am J Surg 191:610-614

39. Balvers K, Van der Horst M, Graumans $M$ et al (2016) Hypothermia as a predictor for mortality in trauma patients at admittance to the Intensive Care Unit. J Emerg Trauma Shock 9:97-102

40. Hsieh TM, Kuo PJ, Hsu SY et al (2018) Effect of hypothermia in the emergency department on the outcome of trauma patients: a cross-sectional analysis. Int J Environ Res Public Health 15:17

41. Ireland $S$, Endacott R, Cameron P et al (2011) The incidence and significance of accidental hypothermia in major trauma-a prospective observational study. Resuscitation 82:300-306

42. Tohme S, Delhumeau C, Zuercher M et al (2014) Prehospital risk factors of mortality and impaired consciousness after severe traumatic brain injury: an epidemiological study. Scand J Trauma Resusc Emerg Med 22:1

43. Gentilello LM, Jurkovich GJ, Stark MS et al (1997) Is hypothermia in the victim of major trauma protective or harmful? A randomized, prospective study. Ann Surg 226:439-447; discussion 447-439

44. Brown DJ, Brugger H, Boyd J et al (2012) Accidental hypothermia. N Engl J Med 367:1930-1938

45. Perlman R, Callum J, Laflamme C et al (2016) A recommended early goal-directed management guideline for the prevention of hypothermia-related transfusion, morbidity, and mortality in severely injured trauma patients. Crit Care 20:107

46. Winkler B, Jenni HJ, Gygax E et al (2016) Minimally invasive extracorporeal circulation resuscitation in hypothermic cardiac arrest. Perfusion 31:489-494

47. Advanced Trauma Life Support, 2019. https://www.facs.org/ quality-programs/trauma/atls

48. TCCC Guidelines for Medical Personnel, 2019, Available from: https://www.naemt.org/docs/default-source/education-docu ments/tccc/tccc-mp-updates-190801/tccc-guidelines-for-medicalpersonnel-190801.pdf?sfvrsn=cc99d692_2

49. Cohen MJ, Christie SA (2017) Coagulopathy of Trauma. Crit Care Clin 33:101-118

50. Chang R, Cardenas JC, Wade CE et al (2016) Advances in the understanding of trauma-induced coagulopathy. Blood 128:1043-1049

51. Hess JR, Brohi K, Dutton RP et al (2008) The coagulopathy of trauma: a review of mechanisms. J Trauma 65:748-754

52. Simmons JW, Powell MF (2016) Acute traumatic coagulopathy: pathophysiology and resuscitation. Br J Anaesth 117:iii31-iii43

53. Maxwell WL, Donnelly S, Sun X et al (1999) Axonal cytoskeletal responses to nondisruptive axonal injury and the short-term effects of posttraumatic hypothermia. J Neurotrauma 16:1225-1234

54. Lustenberger T, Talving P, Kobayashi L et al (2010) Early coagulopathy after isolated severe traumatic brain injury: relationship with hypoperfusion challenged. J Trauma 69:1410-1414

55. De Tanti A, Gasperini G, Rossini M (2005) Paroxysmal episodic hypothalamic instability with hypothermia after traumatic brain injury. Brain Inj 19(14):1277-1283 
56. McHugh GS, Engel DC, Butcher I et al (2007) Prognostic value of secondary insults in traumatic brain injury: results from the IMPACT study. J Neurotrauma 24:287-293

57. Harris OA, Colford JM Jr, Good MC et al (2002) The role of hypothermia in the management of severe brain injury: a metaanalysis. Arch Neurol 59:1077-1083

58. Marmarou A, Lu J, Butcher I et al (2007) IMPACT database of traumatic brain injury: design and description. J Neurotrauma 24:239-250

Publisher's Note Springer Nature remains neutral with regard to jurisdictional claims in published maps and institutional affiliations.

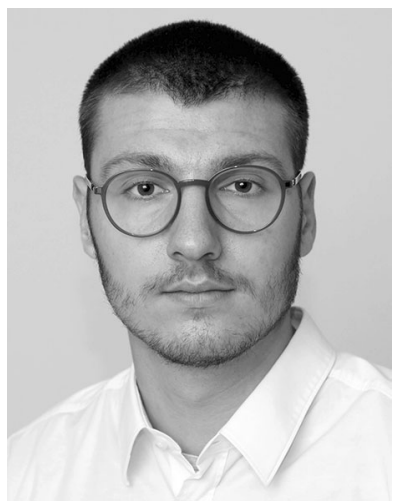

David Rösli is a 6th year medical student at the University of Bern, Switzerland. He completed his bachelor in medicine in 2019 and will graduate from medical school in 2021. Since 2017, David Rösli has been assisting in the $\mathrm{OR}$ at the Inselspital, Bern University Hospital and was able to gain experience in Acute Care Surgery. His main research interests include Acute Care Surgery and Trauma research. In 2019, David Rösli presented the results of this study at the European Congress of Trauma and
Emergency Surgery in Prague, Czech Republic. With the current publication, David Rösli will achieve his MD degree. 